\title{
Elucidating the role of pigment epithelium-derived factor (PEDF) in metabolic PCOS models
}

\author{
Michal Silber ${ }^{1,2,3}$, Irit Miller¹, Hadas Bar-Joseph1, Ido Ben-Ami4,5,* and Ruth Shalgi1,* \\ 'Department of Cell and Developmental Biology, Sackler Faculty of Medicine, Tel Aviv University, Tel Aviv, Israel \\ 2Department of OB/GYN, Meir Medical Center, Kfar Saba, Israel \\ ${ }^{3}$ Sackler Faculty of Medicine, Tel Aviv University, Tel Aviv, Israel \\ 4 IVF and Fertility Unit, Department of OB/GYN, Shaare Zedek Medical Center, Jerusalem, Israel \\ ${ }^{5}$ The Hebrew University Medical School of Jerusalem, Jerusalem, Israel
}

Correspondence should be addressed to R Shalgi: shalgir@tauex.tau.ac.il

*(I Ben-Ami and R Shalgi contributed equally to this work)

\begin{abstract}
PCOS is the most common endocrinopathy in women; associated with obesity and insulin resistance (IR). IR leads to accumulation of advanced-glycation-end-products (AGEs) and their receptor, RAGE. PCOS patients have increased levels of vascular endothelial growth factor (VEGF), interleukin 6/8 (IL-6/8) and anti-Müllerian-hormone (AMH). PEDF is a secreted-glycoprotein known for its anti-angiogenic and antiinflammatory properties. We aimed to elucidate the role of PEDF in the pathogenesis and treatment of PCOS. We used a prenatal PCOS mouse model and fed the female offspring a high-fat diet, inducing metabolic PCOS (met.PCOS) characteristics. Female offspring were divided into three groups: control; met.PCOS; met.PCOS + recombinant PEDF (rPEDF). Met.PCOS mice gained more weight, had elevated serum IL-6 and higher mRNA levels of AMH, PEDF and RAGE in their granulosa cells (GCs) than met. PCOS + rPEDF mice. An in vitro Met.PCOS model in human GCS (KGN) line was induced by prolonged incubation with insulin/AGEs, causing development of IR. Under the same conditions, we observed an elevation of VEGF, IL-6/8 mRNAs, concomitantly with an increase in PEDF $m R N A$, intracellular protein levels, and an elevation of PEDF receptors (PEDF-Rs) mRNA and protein. Simultaneously, a reduction in the secretion of PEDF from GCs, was measured in the medium. The addition of rPEDF (5 nM) activated P38 signaling, implying that PEDF-Rs maintained functionality, and negated AGE-induced elevation of IL-6/8 and VEGF mRNAs. Decreased PEDF secretion may be a major contributor to hyperangiogenesis and chronic inflammation, which lie at the core of PCOS pathogenesis. rPEDF treatment may restore physiological angiogenesis inflammatory balance, thus suggesting a potential therapeutic role in PCOS.
\end{abstract}

Key Words

- PCOS

- PEDF

- insulin resistance

- advanced glycation end-products

- KGN

- IL-6

- IL-8

- VEGF

\section{Introduction}

PCOS is the most common endocrinopathy in women (Azziz et al. 2016). It has become increasingly evident over the years that PCOS is not a single uniform disorder, but rather a group of disorders with the diagnostic criteria serving as the unifying features. Up to two-thirds of PCOS patients exhibit insulin resistance 
(IR; DeUgarte et al. 2005), and up to $76 \%$ are obese (Diamanti-Kandarakis et al. 2012), making PCOS a major metabolic disorder. In addition to obese patients, IR is present in up to $47 \%$ of lean PCOS individuals (Cassar et al. 2016). Evidence suggests that in most cases IR presents with compensatory hyperinsulinemia contributing to hyperandrogenism via stimulation of ovarian androgen secretion and inhibition of hepatic sex hormone-binding globulin production (Teede et al. 2010). The hyperinsulinemia is associated with increased steroidogenesis, dysregulation of differentiation in GCs, and arrest of follicle growth (Franks et al. 2000). IR leads to hyperglycemia, which induces an accelerated production of advanced glycation end-products (AGEs; Friedman 1999), and an upregulation of their receptor, RAGE (Yao \& Brownlee 2010). Accordingly, the levels of AGEs and RAGE were found to be elevated in the ovaries of PCOS patients (Pertynska-Marczewska et al. 2015). The AGE-RAGE axis induces the production of the inflammatory cytokines, interleukins 6/8 (IL-6/8; Nonaka et al. 2017), which aggravate IR (Yamagishi \& Imaizumi 2005). In addition, anti-Müllerian-hormone (AMH) is elevated in PCOS patients, to a point that it was suggested as a biomarker for PCOS diagnosis (Karakas 2017, Sathyapalan et al. 2018). Because of the high levels of cytokines such as IL- 6 and IL-8 in the serum and GCs, PCOS is considered a chronic low-grade inflammatory disorder, associated with obesity, IR, and hyperandrogenism (Schmidt et al. 2014, Shorakae et al. 2015, Peng et al. 2016).

Vascular endothelial growth factor (VEGF) is an important pro-angiogenic factor in normal folliculogenesis, but is also considered a pro-inflammatory agent (Angelo \& Kurzrock 2007). Increased production and secretion of ovarian VEGF have been commonly observed in women with PCOS (reviewed by Di Pietro et al. 2018) and the level of VEGF is higher in the follicular fluid of patients with PCOS than those without (Artini et al. 2009). Overproduction of VEGF and cytokines during the luteal phase after gonadotropin stimulation is thought to be related to the higher risk of ovarian hyperstimulation syndrome (OHSS) in PCOS patients undergoing IVF treatments (Delgado-Rosas et al. 2009).

Pigment epithelium-derived factor (PEDF) is a $50 \mathrm{kDa}$ secreted glycoprotein, and a non-inhibitory member of the serine protease family, first identified as a neurotrophic and anti-angiogenic factor in fetal retinal pigment epithelium (Tombran-Tink et al. 1991). PEDF is a multifactorial protein capable of mediating diverse cellular functions and is known for its antiangiogenic (Simonovic et al. 2001), anti-inflammatory
(Wang et al. 2008), and anti-oxidative (Yamagishi et al. 2005) properties. It is present in most of the tissues in the body, and specifically is highly expressed in the reproductive system (Tombran-Tink et al. 1996). It is also one of the most abundant adipokines in the body (Alvarez-Llamas et al. 2007), exerting its effects in both a paracrine (Loegl et al. 2016) and autocrine (Daubriac et al. 2017) fashion. PEDF has been shown to counteract AGE-mediated IR in a variety of cells (Yoshida et al. 2008, Ishibashi et al. 2013, Seki et al. 2013).

We have previously characterized PEDF in the female reproductive system (Chuderland et al. 2014) where we showed that both rodent and human GCs produce and secrete PEDF in a hormonally dependent manner, mimicking in vitro the peri-ovulatory hormonal changes. This was expressed as an increase in estradiol, upregulation of luteinizing hormone (LH), and/or an increase in progesterone, resulting in the downregulation of PEDF expression and secretion, and increased expression of VEGF and IL-6/8, in accordance with a role as a negative regulator of ovarian angiogenesis (Chuderland et al. 2013a, 2014). We also demonstrated in a OHSS mice model, that the secreted PEDF possesses both anti-angiogenic and antiinflammatory traits, and that treatment with recombinant PEDF (rPEDF) could alleviate OHSS symptoms (Miller et al. 2016). In GCs, PEDF exhibited anti-oxidative activities (Bar-Joseph et al. 2014). Interestingly, the human chorionic gonadotropin (hCG)-induced increased expression of VEGF was mirrored by a decrease in PEDF expression (Chuderland et al. 2013a), while PEDF secretion in vitro was decreased by lysophosphatidic acid (LPA) (Miller et al. 2016). There is now accumulating evidence that specific receptor interactions determine the broad pleiotropy of PEDF biological activities. We selected two well-studied PEDF receptors, the laminin receptor (LR), and adipose triglyceride lipase (ATGL) and evaluated them in the in-vitro model of metabolic PCOS (met.PCOS).

The objective of this study was to explore the metabolic role of PEDF in the pathogenesis and treatment of PCOS.

\section{Materials and methods}

\section{Reagents}

Dulbecco's PBS (dPBS), L-glutamine, penicillin, and streptomycin (Biological Industries, Beit-Ha'emek, Israel); fetal bovine serum (FBS; Invitrogen); insulin (recombinant, rDNA origin, $100 \mathrm{ng} / \mathrm{mL}$, Lilly Pharmaceuticals); AGEs (human glycated albumin, $200 \mu \mathrm{g} / \mathrm{mL}$, Sigma-Aldrich). 


\section{Animals}

ICR female mice (Envigo RMS Limited, Jerusalem, Israel) were housed in temperature- and humidity-controlled rooms at the animal facility of the Sackler Faculty of Medicine, Tel Aviv University, under artificial illumination for $12 \mathrm{~h}$ daily. Food and water were provided ad libitum.

\section{Ethical approval of animal use}

Animal care and all experiments were in accordance with the guidelines of the Institutional Animal Care and Use Committee, Sackler Faculty of Medicine, Tel-Aviv University and were approved by the ethics committee (permit number \#01-15-093).

\section{PCOS mice model}

We followed the animal model of prenatal PCOS induction, described by Roland et al. (2010) and Chuderland et al. (2014). Briefly, the day of the vaginal plug was considered day 1 of gestation. Pregnant mice were injected subcutaneously (SC) on days 16-19 of gestation with $250 \mu \mathrm{g}$ of dehydrotestosterone (DHT; Sigma-Aldrich) dissolved in $100 \mu \mathrm{L}$ propanediol (Sigma-Aldrich) or with the same volume of vehicle (control). Female offspring were weaned at the 21st postnatal day and were fed a regular diet for 3 weeks after weaning. A baseline glucose tolerance test (GTT) was conducted at 6 weeks of age. In order to obtain a metabolic PCOS model (met.PCOS), the offspring of DHT treated pregnant females, were switched to a high-fat diet from this time point (HFD - 60\% calories from fat; Teklad 06414, Envigo RMS Limited). Met.PCOS group mice were randomly divided into two subgroups: $a$. met.PCOS + rPEDF group. $b$. met.PCOS + Tris group; 2. A second GTT was conducted after 10 days of HFD feeding, after which rPEDF treatment was initiated for the met. PCOS +rPEDF group. The met.PCOS+rPEDF mice were treated with rPEDF every third day. Mice in the control and Met.PCOS groups were injected at the same times with a comparable volume of vehicle. Following 8 weeks of treatment, a third GTT was conducted, followed by euthanasia of the mice in order to remove their ovaries and extract the RNA (Fig. 1).

\section{Glucose tolerance test (GTT)}

We followed a well-established protocol (Landsman et al. 2011): mice were injected intraperitoneally (IP) with $20 \%$ dextrose (10 $\mu \mathrm{l} / \mathrm{g}$ body weight; Sigma-Aldrich). Blood was drawn using a tail clip at various time-points after dextrose administration and the glucose level was measured using a standard glucometer (Freestyle Lite, Abbott Diabetes Care, Abbott).

\section{Mouse-primary-granulosa-cells (mpGCs)}

Mouse ovaries were excised and transferred to culture dishes containing serum-free DMEM-F12 (Dulbecco's modified Eagle's medium/Ham F12 1:1, Biological Industries). Follicles were punctured with a $21 \mathrm{G}$ needle, GCs and the cumulus-oocyte complexes were gently squeezed out of the follicles into the culture medium. The GCs were centrifuged (300 $\boldsymbol{g}$ for $5 \mathrm{~min}$ ), seeded in $10 \%$ serum-coated 24-well plates (Nunc, Roskilde, Denmark), and cultured for $4 \mathrm{~h}$ in a humidified incubator at $37^{\circ} \mathrm{C}$ and $5 \% \mathrm{CO}_{2}$ in air.

\section{Cell cultures}

The KGN GCline, purchased from Riken cell bank (Tsukuba, Japan), is derived from a human ovarian GC tumor and expresses typical GC markers. The cells predominately present FSH receptors, making them resemble PCOS GCs (Nishi et al. 2001). KGN cells (<30-line passages) were cultured in DMEM-F12 medium supplemented with
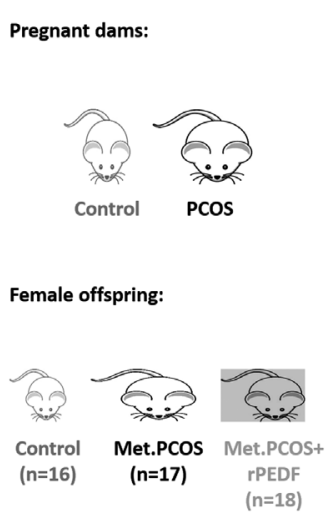
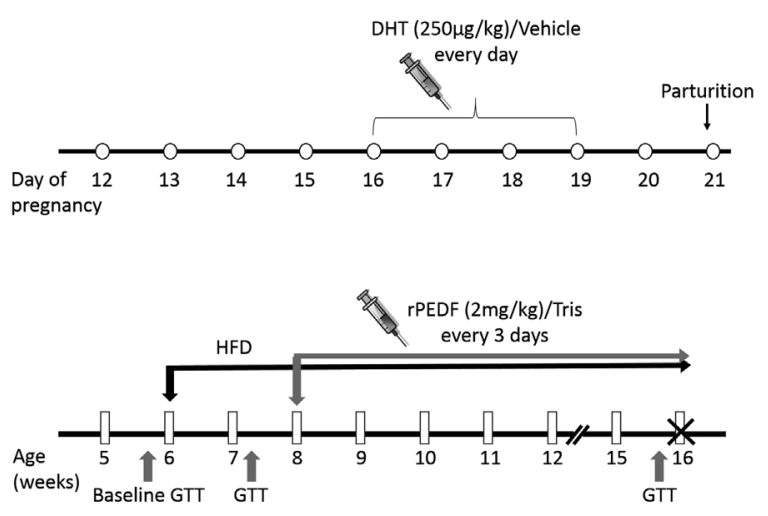

Figure 1

Metabolic PCOS mouse model. 
10\% FBS and $2 \mathrm{mM}$ L-glutamine, penicillin $(10,000 \mathrm{IU} / \mathrm{mL})$, and streptomycin $(10 \mathrm{mg} / \mathrm{mL}$; Biological Industries). Cells were serum-starved in DMEM-F12 (0.1\% FBS, 2 mM L-glutamine, penicillin $(10,000 \mathrm{IU} / \mathrm{mL})$, and streptomycin $(10 \mathrm{mg} / \mathrm{mL}))$ for $16 \mathrm{~h}$ before short stimulations or during prolonged stimulations.

\section{PEDF production}

Human rPEDF (NM_002615.4) was expressed in Escherichia coli BL21, as described previously (Miller et al. 2016). Briefly, recombinant proteins were purified by ion metal affinity chromatography on a Ni-NTA His-Bind resin (Merck) according to the manufacturer's protocol. Eluates with $>90 \%$ purity were dialyzed against Tris buffer ( $\mathrm{pH} 10)$.

\section{RNA isolation}

Total RNA was extracted from GCs with TRIzol reagent (Bio-Tri; BioLab Chemicals, Jerusalem, Israel), according to the manufacturer's instructions, and quantified with the Nano-Drop spectrophotometer (ND-1000; Thermo Scientific). First-strand cDNA was created with a highcapacity cDNA RT kit (Applied Biosystems; 10 ng RNA fractions). Changes in the level of expression of mRNA were detected by quantitative PCR (qPCR) using TaqMan Universal PCR Master Mix (Life Technologies) and $15 \mathrm{ng}$ cDNA and specific primers, on a Step One Plus Real-Time PCR System (Applied Biosystems). Gene expression was detected using specific primers for IL-6 (assay identification (ID) IL-6, Mm00446190_m1; IL6, Rn01410330_m1; IL-6, Hs00985639_m1; IL-8, Hs00174103_m1; PEDF, Mm00441270_m1; PEDF, Hs01106937_m1; VEGF, Mm00437306_m1; VEGF, Hs00900055_m1; Rplp2, Mm00782638_s1; and RPLP0, Hs99999902_m1). Relative expression was calculated using the comparative $\Delta \mathrm{Ct}$.

\section{Enzyme immunoassay}

The level of PEDF in KGN conditioned media and of IL-6 in the mouse serum were determined using the appropriate ELISA kit (R\&D Systems) according to the manufacturer's protocol.

\section{Western blot analysis}

KGN cells were lysed and proteins were extracted and separated as described previously (Miller et al. 2016). Membranes were immunoblotted with
anti-PEDF (1:200, sc-25594, Santa Cruz Biotechnology; or 1:1,000, EPR98269(B), Abcam); anti-pAKT (1:2000, S473, Cell Signaling), anti genAKT (1:10,000, P1601, Sigma-Aldrich); anti-phosphorylated-P38 (1:1,000; M8177; Sigma-Aldrich), anti-general-P38 (1:10,000; M0800; Sigma-Aldrich); anti actin (1:10,000, MAB1501; Millipore); anti-LR antibody (1:500; SC; Santa Cruz Biotechnology), anti-ATGL antibody (1:1,000; SC; Santa Cruz Biotechnology). Horseradish peroxidase-conjugated monoclonal anti-mouse $(1: 10,000)$ or polyclonal antirabbit $(1: 10,000)$ secondary antibodies (Jackson ImmunoResearch Laboratories) were used. Band intensity was quantified by the ImageJ software (NIH).

\section{Statistics}

All statistical analyses were performed by SPSS software (SPSS Inc.). Relative protein, mRNA, and immunoassay results are expressed as mean \pm s.E.M. and compared as appropriate for data distribution. Data normality were assessed using Kolmogorov-Smirnov tests. Normally distributed data were then evaluated by one-way ANOVA followed by Tukey post hoc or parametric two-tailed Student's $t$ test with equal variance ( $t$-test) to evaluate significance. In all instances where raw data were not normally distributed, square root or log transformation were applied to normalize data. Data are shown as percentage normalized to control; $P$ value $<0.05$ was considered statistically significant.

\section{Results}

HFD induces weight gain, impairs glucose tolerance (IGT), and increases IL-6 in an in vivo met.PCOS model

The obesity present in up to $76 \%$ of PCOS patients leads to a dysregulation of metabolic status, and the development of IR. A basal GTT in a random sample from all experimental groups in the in vivo PCOS model, demonstrated normal glucose tolerance (Fig. 2A). However, after 10 days of HFD feeding, a second GTT revealed IGT in all the met.PCOS mice, while the control mice maintained normal glucose tolerance with values similar to the results of the pretreatment GTT $(P<0.01$; Fig. $2 \mathrm{~B})$. HFD feeding caused IGT in both groups of mice (Fig. 2C).

Mice were weighed once a week starting at the initiation of HFD feeding (Fig. 2D). Over this period of time, Met.PCOS mice gained significantly more weight than control mice $(20.2 \pm 4.95 \mathrm{~g}$ and $13.4 \pm 3.48 \mathrm{~g}$, 

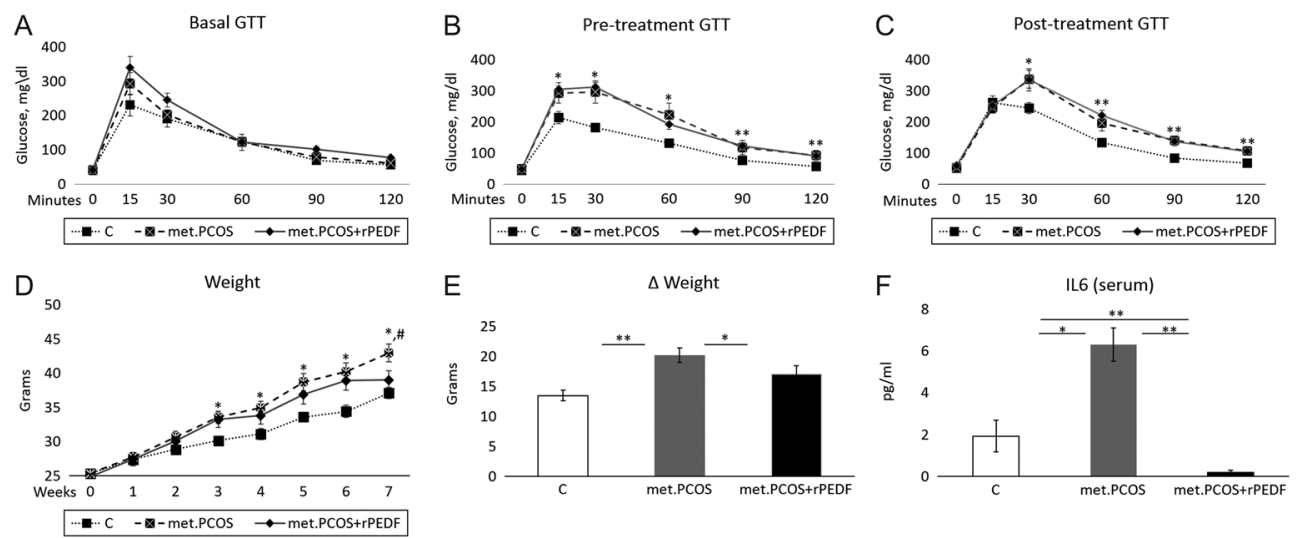

\section{Figure 2}

The effect of rPEDF on Met.PCOS characteristics in an in-vivo PCOS model. (A, B and C) GTT: Mice were injected IP with $20 \%$ dextrose (10 $\mu \mathrm{L} / \mathrm{g}$ body weight) after an overnight fast and blood glucose was measured at various time points up to 120 min post injection. A basal GTT (A) was conducted prior to feeding with HFD, followed by a second GTT (Pre-treatment GT; B) after 10 days of HFD feeding. All HFD-fed mice exhibited IGT, with values that were significantly different from control mice (ANOVA $* P<0.05, * * P<0.01)$. Treatment with rPEDF $(0.05 \mathrm{mg} / \mathrm{kg}$ ) injected SC every 3 days, was initiated after the second GTT (met.PCOS + rPEDF mice; $n=18)$. Control (C; $n=16)$ and Met.PCOS $(n=17)$ mice were injected SC with Tris (rPEDF diluent). Following 8 weeks of treatment, a third GTT was conducted (post-treatment GTT; C). Mice were weighed once a week, starting at the initiation of HFD feeding (D). (E) Delta weight gain. (F) Level of IL-6 in the serum. Sera were collected from each mouse, on the day of euthanization, and subjected to ELISA analysis with antibodies against IL-6. Eight mice in each group. $* P<0.05, * * P<0.01$, significantly different from control mice value, \# no difference between control and met.PCOS + rPEDF (ANOVA).

respectively; $P<0.01$; Fig. 2E). Treatment with rPEDF (met.PCOS + rPEDF mice) resulted in an increase in weight, though significantly lower than that of Met.PCOS mice, treated with Tris $(17.02 \pm 5.8 \mathrm{~g} ; P<0.05$; Fig. $2 \mathrm{E})$.

PCOS is associated with symptoms of chronic mild inflammation, including elevated levels of cytokines (e.g. IL-6). The level of IL-6 is closely associated with obesity in general, and PCOS in particular. The Met.PCOS mice had levels of serum IL- 6 that were three-fold higher than those in control mice $(P<0.01)$. Treatment of Met.PCOS mice with rPEDF restrained this IL-6 elevation to levels that were lower than those in control mice $(P<0.01$; Fig. 2F). We conclude that feeding with HFD induces metabolic characteristics associated with PCOS as manifested by weight gain, IGT, and elevated IL-6, and that rPEDF treatment can attenuate the weight gain and prevent the increase in IL-6.

\section{Primary GCs from met.PCOS mice exhibit elevated RAGE, AMH and PEDF}

The effect of PEDF on IL6, GTT, and weight gain, observed in the Met.PCOS mice model, led us to examine additional parameters. Treatment with rPEDF also attenuated the elevation of RAGE mRNA $(P<0.05)$ seen in Met.PCOS mice $(P<0.05$; Fig. $3 \mathrm{~A})$, and a similar effect was observed in the elevated level of AMH mRNA in the GCs of Met.PCOS mice. In this case, treatment with rPEDF normalized the $80 \%$ elevation seen in the PCOS mice
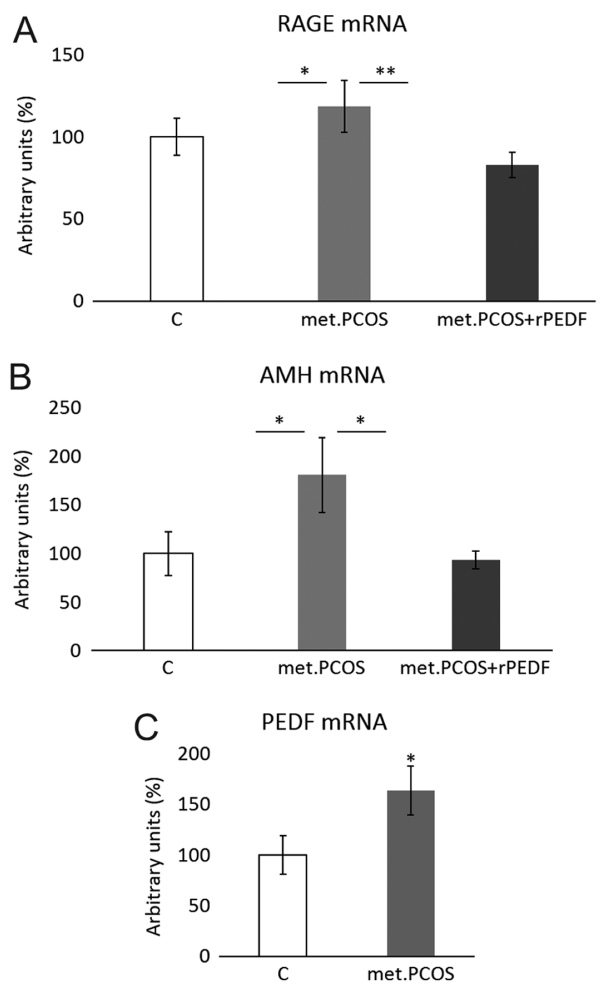

\section{Figure 3}

Elevated mRNA levels of RAGE, AMH and PEDF in mpGCs isolated from Met.PCOS mice. mRNA prepared from mpGCs from individual mice was subjected to quantitative $P C R$ analysis (relative expression), with specific primers for RAGE (A), AMH (B), and PEDF (C), and normalized to the endogenous controls HPRT and RPLP2. Bars represent the mean \pm S.E.M. of relative expression. Eight mice in each group. $* P<0.05, * * P<0.01$ (ANOVA). C, control. 
$(P<0.05$; Fig. 3B). Surprisingly, we found that the level of PEDF mRNA was higher in mpGCs isolated from Met.PCOS mice than in mpGCs isolated from control mice (Fig. 3C).

\section{The in vitro met.PCOS model is associated with elevated inflammatory and angiogenic factors}

To obtain a deeper understanding of the role of PEDF in met.PCOS, we established an in vitro Met.PCOS model using KGN cells. The cells were incubated with insulin or AGEs for 16-48 $\mathrm{h}$, with insulin added for the last 15 min (acute insulin stimulation). The cells were then lysed and the level of AKT phosphorylation, which is one of the important signaling cascades triggered by insulin, was examined. Our results showed that the level of AKT phosphorylation was significantly lower $(P<0.05)$ in cells incubated with insulin for $20 \mathrm{~h}$, or with AGEs for $48 \mathrm{~h}$, than in untreated cells (Fig. 4A, B, C and D), reflecting in vitro IR. Interestingly, the levels of IL-6/8 and VEGF mRNAs were significantly elevated after $16 \mathrm{~h}$ of incubation with insulin or AGEs, preceding the development of IR $(P<0.01$; Fig. 4E). These findings correlate with the in vivo findings, suggesting that this model may serve as a valuable in vitro Met.PCOS model.

\section{PEDF and PEDF-R levels are elevated in the in vitro met.PCOS model}

As expected from the high levels of PEDF seen in the in vivo Met.PCOS mouse model (Fig. 3C), we observed a threefold increase in PEDF mRNA after 16-h incubation with insulin $(P<0.01$; Fig. $5 \mathrm{~A})$ and a 2.5 or 1.5 -fold increase following 12 or $16 \mathrm{~h}$ of incubation with AGEs $(P<0.05$ and $P<0.01$, respectively; Fig. $5 \mathrm{~A})$. Correspondingly, the intracellular level of PEDF protein started to increase after $16 \mathrm{~h}$ and reached a maximum after $20 \mathrm{~h}$ of incubation with both stimulants $(P<0.01 ; \mathrm{Fig}$. $5 \mathrm{C}$ and $\mathrm{D})$. In addition, there was a significant increase in the mRNA levels of the two PEDF receptors, ATGL and LR, after $16 \mathrm{~h}$ of incubation with insulin or AGEs compared to the level in control cells $(P<0.01$ and $P<0.05$; Fig. 5B). Accordingly, the protein levels were also significantly elevated $(P<0.05$ and $P<0.01$; Fig. $5 \mathrm{C}$ and E).

\section{Exogenous rPEDF negates AGEs-induced elevation of cytokine and VEGF mRNA in the in vitro met.PCOS model}

When KGN cells incubated for an extended period with insulin or AGEs (chronic incubation) to simulate PCOS,
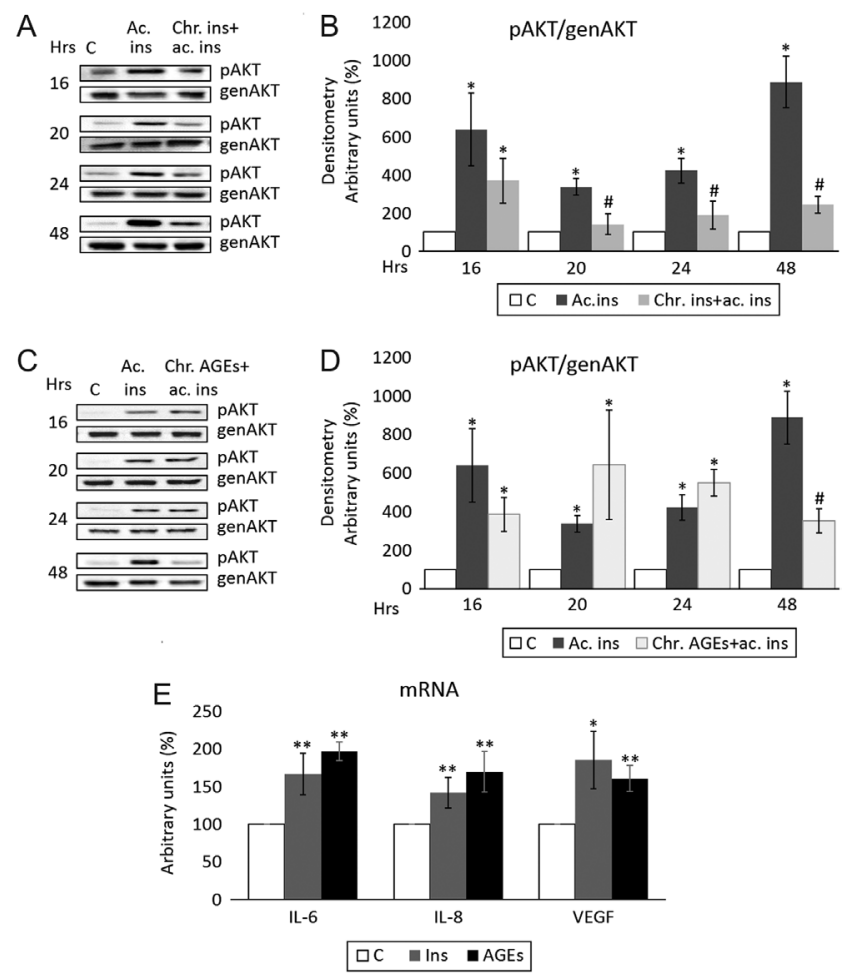

\section{Figure 4}

The in vitro Met.PCOS model has elevated levels of inflammatory, angiogenic and metabolic factors. (A, B, C and D) KGN cells were incubated for $16-48 \mathrm{~h}$ in culture medium containing $0.1-0.5 \% \mathrm{FBS}$ and $100 \mathrm{ng} / \mathrm{mL}$ insulin or $200 \mu \mathrm{g} / \mathrm{mL}$ AGEs (chronic insulin/AGEs; chr.ins./AGEs), and then stimulated with insulin (100 ng/mL for $15 \mathrm{~min}$ - acute insulin; ac. ins.). The cells were then lysed and their proteins analyzed by WB using anti-pAKT (1:2000) and anti-general AKT (genAKT, 1:5000) antibodies. The representative WBs (A and C) depict a 15-min acute stimulation, and a chronic (16-48 h) incubation with insulin/AGEs, before a 15-min acute insulin stimulation (Chr. ins/AGEs + ac. ins). The experiment was repeated at least three times. ( $B$ and $D$ ) WB analysis. Bars represent the mean \pm S.E.M. determined by ImageJ software $(\mathrm{NIH})$.

\#Significantly difference from acute insulin value, $P<0.05$ ( $t$-test). (E) KGN cells were incubated for 16 or $24 \mathrm{~h}$ in culture medium containing $0.1 \%$ FBS and either $100 \mathrm{ng} / \mathrm{mL}$ insulin or $200 \mu \mathrm{g} / \mathrm{mL}$ AGEs. Cells treated with DDW or albumin, served as controls. The corresponding mRNAs were subjected to GPCR analysis with specific primers for IL-6/IL-8 or VEGF, and the values were normalized to the endogenous control, RPLPO. Bars represent mean \pm S.E.M. The experiment was repeated at least three times. $\star P<0.05 ; * \star P<0.01$ (t-test). C, control; Ac.ins, acute insulin; Chr.ins/AGEs, chronic insulin/AGEs; hrs = hours.

were then exposed to rPEDF for 15 min, phosphorylation of P38 was induced to a level similar to that obtained under control conditions (Fig. 6A and B). In order to investigate the anti-inflammatory and anti-angiogenic effects of PEDF in the in vitro Met.PCOS model, we incubated serumstarved KGN cells with AGEs for $24 \mathrm{~h}$, with rPEDF added to the culture medium for the last $6 \mathrm{~h}$ of incubation. While exposure to AGEs significantly enhanced the expression of IL-6/8 and VEGF mRNAs ( $P<0.01$; Fig. 6C), this increase was completely abolished by co-stimulation with rPEDF 

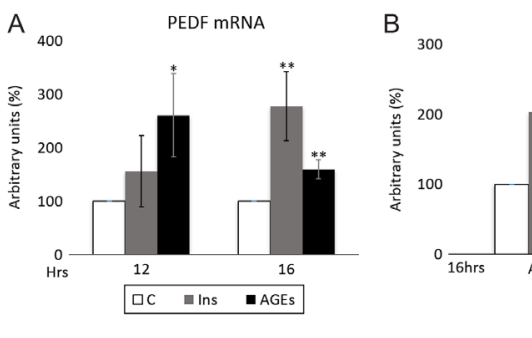

PEDF-R mRNA
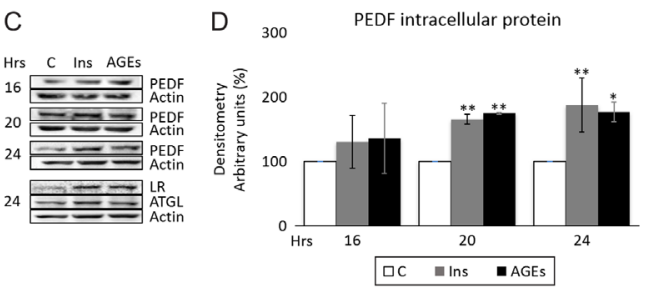

E 300 PEDF-R protein

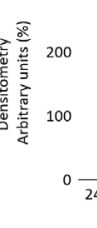

Figure 5

PEDF mRNA, intracellular protein as well as PEDF-R mRNA and protein are elevated in the in vitro Met.PCOS model. (A and B) KGN cells were incubated for 12-16 $\mathrm{h}$ in culture medium containing $0.1 \% \mathrm{FBS}$ and either $100 \mathrm{ng} / \mathrm{mL}$ insulin or $200 \mu \mathrm{g} / \mathrm{mL}$ AGEs. Cells treated with DDW or albumin served as controls. The corresponding mRNAs were subjected to qPCR analysis, with specific primers for PEDF (A), ATGL, LR (B) and normalized to the endogenous control, RPLPO. Bars represent the mean \pm S.E.M. of relative expression. (C) Representative WB. KGN cells were incubated for $16-24 \mathrm{~h}$ in culture medium containing $0.1 \%$ FBS and $100 \mathrm{ng} / \mathrm{mL}$ insulin or $200 \mu \mathrm{g} / \mathrm{mL}$ AGEs. The cells were then lysed and their proteins analyzed by WB using anti-PEDF (1:2000), anti-ATGL (1:1000), anti-LR (1:200), and anti-actin (1:10000) antibodies. (D and E) WB analysis. Bars represent the mean \pm S.E.M. of relative expression evaluated by Image software $(\mathrm{NIH})$. All experiments were repeated at least three times. ${ }^{*} P<0.05 ; * \star P<0.01$ (t-test).

C, control; Ins, insulin; hrs, hours.
$(P<0.05 ;$ Fig. 6C). From these results, we conclude that the PEDF receptors remained functional even under the in vitro Met.PCOS conditions and that the increases in PEDF, IL-6/8 and VEGF cannot be a consequence of a malfunction of PEDF-Rs. Unlike the situation with AGEs, co-stimulation with rPEDF and insulin had no effect on the PCOS like increases in IL-6/8 and VEGF mRNA (data not shown).

\section{PEDF secretion is inhibited in the in vitro met.PCOS model}

Sixteen hours of incubation, with insulin or AGEs, significantly reduced PEDF secretion by $20 \%$ or $40 \%$ respectively $(P<0.01)$. This reduction increased to $50 \%$ after 24-h incubation with either insulin or AGEs $(P<0.01$; Fig. 7). These findings suggest that hyperinsulinemia and exposure to AGEs impair PEDF secretion, which may explain the elevation of VEGF and cytokines observed in the Met.PCOS in vitro model.

\section{Discussion}

PCOS is a heterogeneous condition and can exhibit phenotypes with varying levels of underlying hyperandrogenism, menstrual disorders, and polycystic ovarian morphology. Although the PCOS diagnostic criteria do not currently include IR, it is widely acknowledged that IR plays a key role in the pathophysiology of PCOS (Cassar et al. 2016). Importantly, the metabolic aspects of the syndrome have a profound influence on the patient's well-being and overall health. Accumulating evidence indicates that low-grade chronic inflammation and angiogenesis may also contribute to this syndrome (Goodarzi et al. 2011, Nandi et al. 2014, Azziz et al. 2016), although the relationship between the factors is not well understood. The aim of this study was to investigate the role of the anti-inflammatory, anti-angiogenic factor, PEDF, in the metabolic aspects of PCOS.

For this purpose, we induced the metabolic characteristics of IR in an in vivo PCOS mouse model and in in vitro GC cultures. PCOS mice of the prenatal DHT model, used previously in our lab (Chuderland et al. 2014), present a-cyclicity or irregular cycles, oligo-ovulation or an-ovulation, decreased progesterone and follicular atresia. They do not exhibit accelerated weight gain or glucose intolerance; so in order to reach metabolic parameters of PCOS they were also fed an HFD, as described by Lai et al. (2014). The mice exhibited IR, dyslipidemia and hepatic steatosis (Lai et al. 2014, Skaznik-Wikiel et al. 2016). As expected, the Met.PCOS mice were obese and glucose intolerant; they exhibited elevated RAGE mRNA in mpGCs. To ensure that our mice were indeed PCOS-like, we used a surrogate marker, namely the timing of vaginal opening (a marker of puberty onset), and found that vaginal opening in PCOS-induced mice was significantly delayed as compared to control mice. Interestingly, in rPEDFtreated PCOS mice, vaginal opening occurred significantly earlier than in non-treated PCOS mice (data not shown). Once the mice developed IGT, we started treatment with rPEDF, which decreased the expression of RAGE mRNA 
A

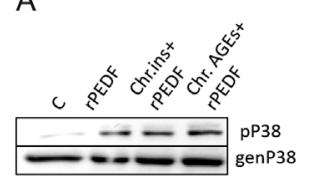

B 250
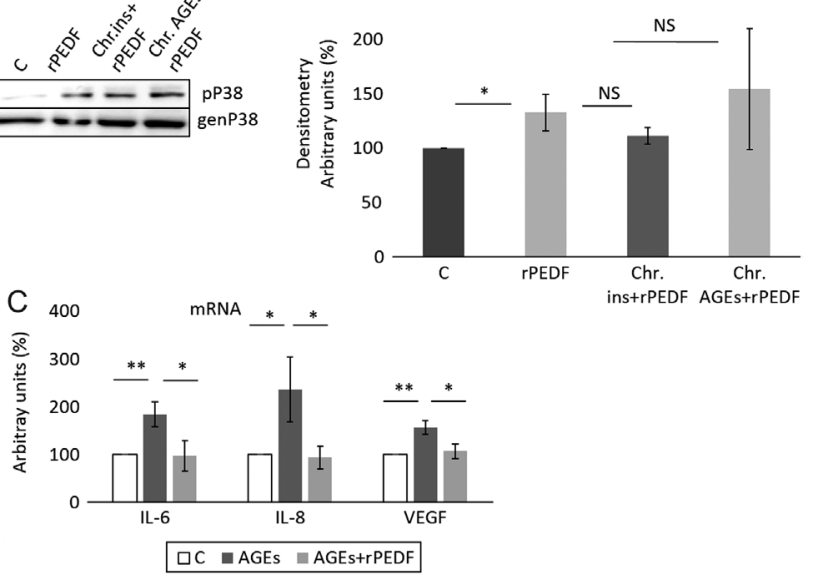

Figure 6

rPEDF stimulates KGN cells in the in vitro Met.PCOS model and negates AGEs-induced cytokine and VEGF mRNA elevation. (A) Representative WB. KGN cells were incubated for $24 \mathrm{~h}$ in culture medium containing $0.1 \% \mathrm{FBS}$ and either $100 \mathrm{ng} / \mathrm{mL}$ insulin or $200 \mu \mathrm{g} / \mathrm{mL}$ AGEs. Cells treated with DDW or albumin served as control. For the last 15 min of incubation, rPEDF ( $5 \mathrm{nM}$ ) was added to the culture medium of untreated cells (rPEDF) and to the medium of cells incubated with insulin or AGEs; Tris was added to control cultures. Cells were lysed and their proteins analyzed by WB using anti-phosphorylated-P38 (pP38, 1:1000) and anti-general-P38 (genP38, 1:10,000). (B) WB analysis. Bars represent the mean \pm s.E.M. of the relative expression evaluated by ImageJ software (NIH). (C) KGN cells were incubated for $24 \mathrm{~h}$ in culture medium containing $0.1 \% \mathrm{FBS}$ and $200 \mu \mathrm{g} / \mathrm{mL}$ AGEs. Cells treated with albumin served as control. rPEDF $(5 \mathrm{nM})$ was added to the culture medium for the last $6 \mathrm{~h}$ of incubation. The corresponding mRNAs were subjected to qPCR analysis, with specific primers for IL-6, IL-8, and VEGF and normalized to the endogenous control, RPLPO. Bars represent the mean \pm S.E.M. of relative expression. All experiments were repeated at least three times. ${ }^{*} P<0.05$; $* * P<0.01$ (t-test). C, control; Chr.ins, chronic insulin; Chr.AGEs, chronic AGEs.

in the mpGCs. These results are consisted with previously published studies, which showed that rPEDF negated the AGE-RAGE axis in a variety of cells in vitro (Yamagishi et al. 2007, Maeda et al. 2011, Ishibashi et al. 2013). In addition, our results showed that treatment with rPEDF attenuated the accelerated weight gain of HFD-fed PCOS mice, a recently published effect of rPEDF (Chen et al. 2019). Total visceral fat, s.c. fat weight, adipocyte area in the white adipose tissue and the number of lipid droplets in the brown adipose tissue were all reduced in mice that received additional PEDF compared to HFD-treated mice, suggesting that the anti-obesity effects of PEDF are mediated by adipogenesis inhibition and lipolysis induction. In our study, despite the positive effects of rPEDF on weight gain, RAGE and inflammatory status, there was no effect on IGT. This finding may be attributed to (a) an insufficient dose of $\mathrm{rPEDF}$, and/or (b) an insufficient treatment duration, and/or (c) mouse strain. Using the prenatal DHT-PCOS model previously, we found decreased PEDF mRNA in
A

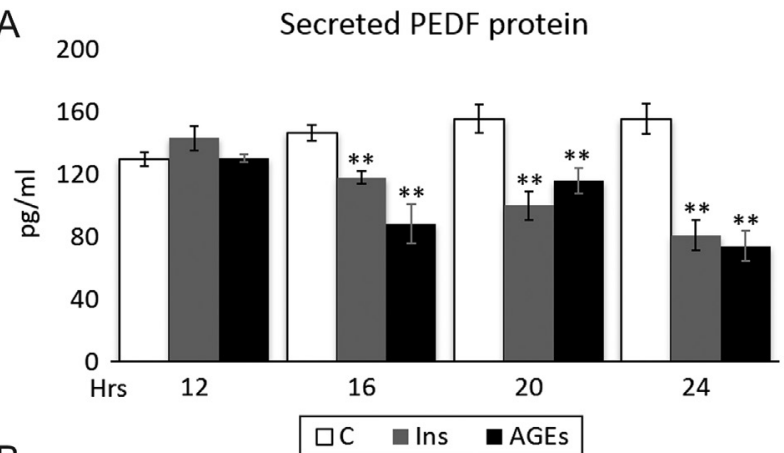

B

\begin{tabular}{|c|c|c|c|}
\hline Hrs & \multicolumn{1}{|c}{ C } & \multicolumn{1}{c|}{ Ins } & \multicolumn{1}{c|}{ AGEs } \\
\hline 12 & $129.5 \pm 9.7$ & $142.8 \pm 17.4$ & $129.8 \pm 5.6$ \\
\hline 16 & $146.0 \pm 6.0$ & $117.6 \pm 9.5$ & $88.0 \pm 20.2$ \\
\hline 20 & $155.0 \pm 19.5$ & $99.5 \pm 10.3$ & $115.3 \pm 13.5$ \\
\hline 24 & $155.0 \pm 30.3$ & $80.6 \pm 14.5$ & $73.6 \pm 19.0$ \\
\hline
\end{tabular}

\section{Figure 7}

PEDF secretion is reduced in the in vitro PCOS model. KGN cells were incubated for $12-24 \mathrm{~h}$ in culture medium containing $0.1 \%$ FBS and either $100 \mathrm{ng} / \mathrm{mL}$ insulin or $200 \mu \mathrm{g} / \mathrm{mL}$ AGEs. Cells treated with DDW or albumin served as control. Media were collected and subjected to ELISA analysis with antibodies against PEDF. (A) Graphic illustration of the raw data $(\mathrm{pg} / \mathrm{mL})$; mean \pm s.D. presented in (B). The experiment was repeated at least five times. ${ }^{*} P<0.05 ;{ }^{*} P<0.01$ ( $t$-test). $C$, control; Ins, insulin; hrs, hours.

whole ovaries of PCOS mice compared to control mice (Chuderland et al. 2014). However, in the Met.PCOS model, we surprisingly found an increase in the PEDF mRNA in the mpGCs, an elevation that may be attributed to the metabolic effects of the HFD. We did not measure the level of PEDF mRNA in the met.PCOS+rPEDF group, so as to refrain from confounding the results.

The Met.PCOS mice in the present study exhibited known PCOS characteristics, with respect to the typical metabolic traits and also the elevated levels of IL-6 (Deligeoroglou et al. 2012) in the serum and AMH in GCs (Sathyapalan et al. 2018). However, similar to the results observed in the PCOS-mice, rPEDF treatment lowered IL-6 and AMH in the GCs of the Met.PCOS to control levels suggesting that the effects of PEDF are common to both models. Low-grade chronic inflammation represents one of the potential links between hyperandrogenism, insulin resistance, obesity, and the long-term consequences of PCOS, such as type 2 diabetes and vascular complications (Repaci et al. 2011). Decreasing IL-6 may benefit the overall metabolic status of the PCOS patient. Since AMH inhibits the FSH-dependent follicle recruitment (Dewailly et al. 2016), our results suggest that PEDF may play an essential role in the sensitivity to FSH of follicles in which AMH is expressed (Durlinger et al. 2001). 
In order to better understand the role of PEDF in met.PCOS, we established an in-vitro Met.PCOS model, in which the KGN GC line was exposed to insulin and AGEs to induce IR. Prolonged exposure to insulin was previously shown to reduce the phosphorylation of insulin-induced AKT in muscle biopsies from PCOS patients compared with muscle tissue from control women (Højlund et al. 2008). A decrease in AKT phosphorylation following prolonged insulin incubation was also previously shown in luteinized human pGCs (Iwase et al. 2009). The phosphorylation of AKT in our in vitro Met.PCOS model was similarly significantly reduced, while the levels of IL-6 and IL-8 mRNA were elevated. These results are consistent with our previous study in PCOS mice model (Miller et al. 2016) and with past studies that demonstrated elevated levels of IL-6 and IL-8 mRNA in the granulosa-lutein cells (Schmidt et al. 2014) and follicular fluid (Artimani et al. 2018) of PCOS patients. The mRNA of VEGF was also significantly elevated in our model, and this is comparable to studies in human subjects with PCOS, which showed that the high levels of VEGF seen in the serum were derived from GCs (Agrawal et al. 2002).

Akin to our findings in the in vivo Met.PCOS model, there was an increase in both PEDF mRNA and protein in the in vitro Met.PCOS model. As already discussed for the DHT mouse model, this increase is in contrast to in vitro findings in an androgenic PCOS model, in which DHT led to elevation of IL-6 mRNA but decreased PEDF mRNA (I Miller, H Bar-Joseph, L Nemerovsky, I Ben-Ami and R Shalgi, unpublished observations). The discrepancies may be explained by the differences between the models, and may reflect the clinical diversity in the most prevalent PCOS aspect in women.
In the current Met.PCOS model, we found an elevation in both the mRNA and protein of PEDF receptor. The question of why there should be a concurrent increase in both intracellular PEDF and PEDF-R led us to examine the functionality of the PEDF-Rs. Since exogenous PEDF activated phosphorylation of P38 and lowered IL-6/8 and VEGF mRNA, it was apparent that the PEDF-R maintained functionality, but an examination in the in vitro Met.PCOS model, indicated inhibition of PEDF secretion accompanied by a consequent PEDF accumulation in the cells. This is the first time that a decrease in PEDF secretion under metabolic conditions has been reported in any cell type. The dramatic decrease in PEDF secretion observed $24 \mathrm{~h}$ after incubation with insulin or AGEs, may explain the increases in PEDF-R mRNA and protein as well as the upregulation of VEGF and IL-6/8 mRNAs.

The precise mechanism of PEDF secretion is yet to be elucidated. PEDF is secreted from adipocytes; adipocytederived PEDF is the major component of PEDF in the serum. We examined locally its secretion from GCs. A number of factors have been shown to influence PEDF secretion from GCs. Stimulation of GCs with increasing doses of estrogen caused a gradual decrease in PEDF secretion, whereas stimulation with progesterone caused an abrupt decrease in PEDF secretion. Similarly, hCG caused downregulation of PEDF (Chuderland et al. 2013a, Bar-Joseph et al. 2016), but no explanation toward the mechanism of the decreased secretion is offered, even in other systems: hyperglycemia decreases PEDF secretion in primary mesangial cells (Wang et al. 2005); and PEDF secretion is decreased in advanced breast cancer (Cai et al. 2006). Evidence toward the understanding of how
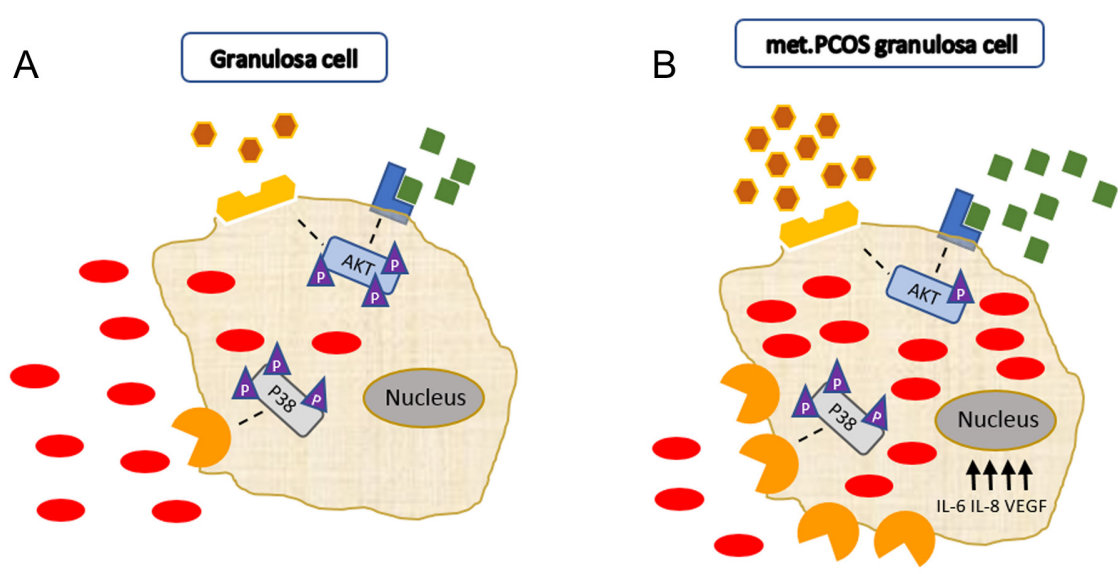

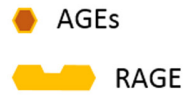

PEDF

PEDF-R
Figure 8

The granulosa cell under normal conditions and in PCOS. (A) A GC under physiological conditions. (B) Met.PCOS GC, hyperinsulinism and hyper AGEs lead to a decrease in secretion of PEDF, PEDF-Rs are elevated and remain functional, extracellular PEDF is diminished and PEDF accumulates in the cell, synthesis of IL-6/8 and VEGF mRNA is increased, and phosphorylation of AKT is diminished. A full colour version of this figure is available at https://doi.org/10.1530/JOE-19-0297. https://joe.bioscientifica.com https://doi.org/10.1530/JOE-19-0297 (c) 2020 Society for Endocrinology Published by Bioscientifica Ltd. Printed in Great Britain 
PEDF secretion may be impaired comes from two sources: back in 2003, it was shown that truncated forms of PEDF could not be secreted (Shao et al. 2003), hinting that blocking PEDF secretion involves changing its structure or influencing the endoplasmic reticulum. It was also shown that GLUT1 activity contributes to the impairment of PEDF secretion in RPE cells (Calado et al. 2016), indicating that specific signaling makes secretion of PEDF possible, and that this signaling is perhaps blocked by insulin and AGEs. Our preliminary results indicate that Brefeldin A, a Golgi apparatus inhibitor, blocks about $50 \%$ of PEDF secretion, implying that PEDF is secreted both consecutively and in a regulated fashion (data not shown). More elaborated studies, which are beyond the scope of the present one, are needed to clarify the mechanism of PEDF secretion.

Our results lead us to propose a mechanism of action for PEDF in GCs, whereby the normal physiological balance between intracellular PEDF, extracellular PEDF and IL-6/8 and VEGF mRNA (Fig. 8A) is disrupted in the Met.PCOS GCs when IR develops. Under these conditions, PEDF secretion is reduced and the protein accumulates inside the cell causing an increase in IL-6/8 and VEGF mRNA (Fig. 8B).

Previous studies from our lab showed that rPEDF treatment can prevent and treat endometriosis lesions (Chuderland et al. 2013c) and OHSS (Miller et al. 2015, 2016). The current findings demonstrate that rPEDF may have a beneficial effect in met.PCOS, by reducing the excessive expression of cytokines, VEGF, AMH, and RAGE in the ovary. These results suggest a therapeutic potential for PEDF with the ability to regulate the impaired inflammatory-angiogenic-hormonal ovarian balance known to play a role in PCOS pathophysiology and restore the physiological ovarian balance. On a broader aspect, our finding that the extracellular secretion of PEDF is disrupted under metabolic conditions and the demonstration that the situation may be rectified by treatment with $\mathrm{rPEDF}$ is a novel outcome, which may be relevant to other organs that are affected by IR.

\section{Declaration of interest}

I B-A and R S are inventors of US Patent 8,962,561; 9,901,619 and Europe 2498806. The other authors have nothing to disclose.

\section{Funding}

This work was partially supported by a grant from the Israel Science Foundation (No. 1934/13 to I B-A).

(C) 2020 Society for Endocrinology Published by Bioscientifica Ltd. Printed in Great Britain

\section{Author contribution statement}

This work was performed in partial fulfillment of the requirements for a PhD degree by M S at the Sackler Faculty of Medicine, Tel Aviv University, Tel-Aviv, Israel. M S designed the study and carried out the experimental work, analyzed the data, and drafted the manuscript. I M assisted in designing the study and preparing the manuscript. H B-J assisted in the in vivo experiments. I B-A conceived the study, participated in analyzing and discussing the results, and helped in drafting the manuscript. R S conceived the study, participated in its design and coordination, helped to draft the manuscript, and supervised the study. I B-A and R S: contributed equally to this work.

\section{Acknowledgements}

The authors thank Prof. T Yanase and Prof. Y Nishi (Department of Endocrinology and Diabetes Mellitus, School of Medicine, Fukuoka University, Fukuoka, Japan) for providing the KGN cells.

\section{References}

Agrawal R, Jacobs H, Payne N \& Conway G 2002 Concentration of vascular endothelial growth factor released by cultured human luteinized granulosa cells is higher in women with polycystic ovaries than in women with normal ovaries. Fertility and Sterility $\mathbf{7 8}$ 1164-1169. (https://doi.org/10.1016/S0015-0282(02)04242-5)

Alvarez-Llamas G, Szalowska E, de Vries MP, Weening D, Landman K, Hoek A, Wolffenbuttel BHR, Roelofsen H \& Vonk RJ 2007 Characterization of the human visceral adipose tissue secretome. Molecular and Cellular Proteomics 6 589-600. (https://doi.org/10.1074/ mcp.M600265-MCP200)

Angelo LS \& Kurzrock R 2007 Vascular endothelial growth factor and its relationship to inflammatory mediators. Clinical Cancer Research 13 2825-2830. (https://doi.org/10.1158/1078-0432.CCR-06-2416)

Artimani T, Karimi J, Mehdizadeh M, Yavangi M, Khanlarzadeh E, Ghorbani M, Asadi S \& Kheiripour N 2018 Evaluation of pro-oxidantantioxidant balance (PAB) and its association with inflammatory cytokines in polycystic ovary syndrome (PCOS). Gynecological Endocrinology 34 148-152. (https://doi.org/10.1080/09513590.2017. 1371691)

Artini PG, Ruggiero M, Parisen Toldin MR, Monteleone P, Monti M, Cela V \& Genazzani AR 2009 Vascular endothelial growth factor and its soluble receptor in patients with polycystic ovary syndrome undergoing IVF. Human Fertility 12 40-44. (https://doi. org/10.1080/14647270802621358)

Azziz R, Carmina E, Chen Z, Dunaif A, Laven JSE, Legro RS, Lizneva D, Natterson-Horowtiz B, Teede HJ \& Yildiz BO 2016 Polycystic ovary syndrome. Nature Reviews: Disease Primers 2 16057. (https://doi. org/10.1038/nrdp.2016.57)

Bar-Joseph H, Ben-Ami I, Ron-El R, Shalgi R \& Chuderland D 2014 Pigment epithelium-derived factor exerts antioxidative effects in granulosa cells. Fertility and Sterility 102 891.e3-898.e3. (https://doi. org/10.1016/j.fertnstert.2014.06.012)

Bar-Joseph H, Ben-Ami I, Ron-El R, Shalgi R \& Chuderland D 2016 Pigment epithelium-derived factor regulation by human chorionic gonadotropin in granulosa cells. Reproduction 151 179-185. (https:// doi.org/10.1530/REP-15-0478)

Cai J, Parr C, Watkins G, Jiang WG \& Boulton M 2006 Decreased pigment epithelium-derived factor expression in human breast cancer progression. Clinical Cancer Research 12 3510-3517. (https://doi. org/10.1158/1078-0432.CCR-06-0094)

Calado SM, Alves LS, Simão S \& Silva GA 2016 GLUT1 activity contributes to the impairment of PEDF secretion by the RPE. Molecular Vision 22 761-770. 
Cassar S, Misso ML, Hopkins WG, Shaw CS, Teede HJ \& Stepto NK 2016 Insulin resistance in polycystic ovary syndrome: a systematic review and meta-analysis of euglycaemic-hyperinsulinaemic clamp studies. Human Reproduction 31 2619-2631. (https://doi.org/10.1093/humrep/ dew243)

Chen CC, Lee TY, Leu YL \& Wang SH 2019 Pigment epithelium-derived factor inhibits adipogenesis in 3T3-L1 adipocytes and protects against high-fat diet-induced obesity and metabolic disorders in mice. Translational Research 210 26-42. (https://doi.org/10.1016/j. trsl.2019.04.006)

Chuderland D, Ben-Ami I, Kaplan-Kraicer R, Grossman H, Ron-El R \& Shalgi R $2013 a$ The role of pigment epithelium-derived factor in the pathophysiology and treatment of ovarian hyperstimulation syndrome in mice. Journal of Clinical Endocrinology and Metabolism 98 E258-E266. (https://doi.org/10.1210/jc.2012-3037)

Chuderland D, Ben-Ami I, Kaplan-Kraicer R, Grossman H, Komsky A, Satchi-Fainaro R, Eldar-Boock A, Ron-El R \& Shalgi R 2013b Hormonal regulation of pigment epithelium-derived factor (PEDF) in granulosa cells. Molecular Human Reproduction 19 72-81. (https://doi. org/10.1093/molehr/gas046)

Chuderland D, Hasky N, Ben-Ami I, Kaplan-Kraicer R, Grossman H \& Shalgi R 2013c A physiological approach for treating endometriosis by recombinant pigment epithelium-derived factor (PEDF). Human Reproduction 28 1626-1634. (https://doi.org/10.1093/humrep/det027)

Chuderland D, Ben-Ami I, Bar-Joseph H \& Shalgi R 2014 Role of pigment epithelium-derived factor in the reproductive system. Reproduction 148 R53-R61. (https://doi.org/10.1530/REP-14-0251)

Daubriac J, Pandya UM, Huang KT, Pavlides SC, Gama P, Blank SV, Shukla P, Crawford SE \& Gold LI 2017 Hormonal and growth regulation of epithelial and stromal cells From the normal and malignant endometrium by pigment epithelium-derived factor. Endocrinology 158 2754-2773. (https://doi.org/10.1210/en.2017-00028)

Delgado-Rosas F, Gaytan M, Morales C, Gomez R \& Gaytan F 2009 Superficial ovarian cortex vascularization is inversely related to the follicle reserve in normal cycling ovaries and is increased in polycystic ovary syndrome. Human Reproduction 24 1142-1151. (https://doi. org/10.1093/humrep/dep008)

Deligeoroglou E, Vrachnis N, Athanasopoulos N, Iliodromiti Z, Sifakis S, Iliodromiti S, Siristatidis C \& Creatsas G 2012 Mediators of chronic inflammation in polycystic ovarian syndrome. Gynecological Endocrinology 28 974-978. (https://doi.org/10.3109/09513590.2012. 683082)

DeUgarte CM, Bartolucci AA \& Azziz R 2005 Prevalence of insulin resistance in the polycystic ovary syndrome using the homeostasis model assessment. Fertility and Sterility 83 1454-1460. (https://doi. org/10.1016/j.fertnstert.2004.11.070)

Dewailly D, Robin G, Peigne M, Decanter C, Pigny P \& Catteau-Jonard S 2016 Interactions between androgens, FSH, anti-Mullerian hormone and estradiol during folliculogenesis in the human normal and polycystic ovary. Human Reproduction Update 22 709-724. (https://doi. org/10.1093/humupd/dmw027)

Di Pietro M, Pascuali N, Parborell F \& Abramovich D 2018 Ovarian angiogenesis in polycystic ovary syndrome. Reproduction $\mathbf{1 5 5}$ R199-R209. (https://doi.org/10.1530/REP-17-0597)

Diamanti-Kandarakis E, Christakou C \& Marinakis E 2012 Phenotypes and enviromental factors: their influence in PCOS. Current Pharmaceutical Design 18 270-282. (https://doi. org/10.2174/138161212799040457)

Durlinger AL, Gruijters MJ, Kramer P, Karels B, Kumar TR, Matzuk MM, Rose UM, de Jong FH, Uilenbroek JT, Grootegoed JA, et al. 2001 Anti-Müllerian hormone attenuates the effects of FSH on follicle development in the mouse ovary. Endocrinology 142 4891-4899. (https://doi.org/10.1210/endo.142.11.8486)

Franks S, Mason H \& Willis D 2000 Follicular dynamics in the polycystic ovary syndrome. Molecular and Cellular Endocrinology 163 49-52. (https://doi.org/10.1016/S0303-7207(99)00239-7)
Friedman EA 1999 Advanced glycosylated end products and hyperglycemia in the pathogenesis of diabetic complications. Diabetes Care 22 (Supplement 2) B65-B71.

Goodarzi MO, Dumesic DA, Chazenbalk G \& Azziz R 2011 Polycystic ovary syndrome: etiology, pathogenesis and diagnosis. Nature Reviews: Endocrinology 7 219-231. (https://doi.org/10.1038/nrendo.2010.217)

Højlund K, Glintborg D, Andersen NR, Birk JB, Treebak JT, Frøsig C, Beck-Nielsen H \& Wojtaszewski JFP 2008 Impaired insulin-stimulated phosphorylation of Akt and AS160 in skeletal muscle of women with polycystic ovary syndrome is reversed by pioglitazone treatment. Diabetes 57 357-366. (https://doi.org/10.2337/db07-0706)

Ishibashi Y, Matsui T, Ohta K, Tanoue R, Takeuchi M, Asanuma K, Fukami K, Okuda S, Nakamura K \& Yamagishi S 2013 PEDF inhibits AGE-induced podocyte apoptosis via PPAR-gamma activation. Microvascular Research 85 54-58. (https://doi.org/10.1016/j. mvr.2012.10.007)

Iwase A, Goto M, Harata T, Takigawa S, Nakahara T, Suzuki K, Manabe S \& Kikkawa F 2009 Insulin attenuates the insulin-like growth factor-I (IGF-I)-Akt pathway, not IGF-I-extracellularly regulated kinase pathway, in luteinized granulosa cells with an increase in PTEN. Journal of Clinical Endocrinology and Metabolism 94 2184-2191. (https://doi.org/10.1210/jc.2008-1948)

Karakas SE 2017 New biomarkers for diagnosis and management of polycystic ovary syndrome. Clinica Chimica Acta 471 248-253. (https://doi.org/10.1016/j.cca.2017.06.009)

Lai H, Jia X, Yu Q, Zhang C, Qiao J, Guan Y \& Kang J 2014 High-fat diet induces significant metabolic disorders in a mouse model of polycystic ovary syndrome. Biology of Reproduction 91 127-127. (https://doi.org/10.1095/biolreprod.114.120063)

Landsman L, Parent A \& Hebrok M 2011 Elevated Hedgehog/Gli signaling causes $\beta$-cell dedifferentiation in mice. PNAS 108 17010-17015. (https://doi.org/10.1073/pnas.1105404108)

Loegl J, Nussbaumer E, Hiden U, Majali-Martinez A, Ghaffari-TabriziWizy N, Cvitic S, Lang I, Desoye G \& Huppertz B 2016 Pigment epithelium-derived factor (PEDF): a novel trophoblast-derived factor limiting feto-placental angiogenesis in late pregnancy. Angiogenesis 19 373-388. (https://doi.org/10.1007/s10456-016-9513-x)

Maeda S, Matsui T, Takeuchi M, Yoshida Y, Yamakawa R, Fukami K \& Yamagishi S 2011 Pigment epithelium-derived factor (PEDF) inhibits proximal tubular cell injury in early diabetic nephropathy by suppressing advanced glycation end products (AGEs)-receptor (RAGE) axis. Pharmacological Research 63 241-248. (https://doi.org/10.1016/j. phrs.2010.11.008)

Miller I, Chuderland D, Ron-El R, Shalgi R \& Ben-Ami I 2015 GnRH agonist triggering modulates PEDF to VEGF ratio inversely to hCG in granulosa cells. Journal of Clinical Endocrinology and Metabolism $\mathbf{1 0 0}$ E1428-E1436. (https://doi.org/10.1210/jc.2015-2312)

Miller I, Chuderland D, Grossman H, Ron-El R, Ben-Ami I \& Shalgi R 2016 The dual role of PEDF in the pathogenesis of OHSS: negating both angiogenic and inflammatory pathways. Journal of Clinical Endocrinology and Metabolism 101 4699-4709. (https://doi. org/10.1210/jc.2016-1744)

Nandi A, Chen Z, Patel R \& Poretsky L 2014 Polycystic ovary syndrome. Endocrinology and Metabolism Clinics of North America 43 123-147. (https://doi.org/10.1016/j.ecl.2013.10.003)

Nishi Y, Yanase T, Mu Y, Oba K, Ichino I, Saito M, Nomura M, Mukasa C, Okabe T, Goto K, et al. 2001 Establishment and characterization of a steroidogenic human granulosa-like tumor cell line, KGN, that expresses functional follicle-stimulating hormone receptor. Endocrinology 142 437-445. (https://doi.org/10.1210/endo.142.1.7862)

Nonaka K, Kajiura Y, Bando M, Sakamoto E, Inagaki Y, Lew JH, Naruishi K, Ikuta T, Yoshida K, Kobayashi T, et al. 2017 Advanced glycation end-products increase IL- 6 and ICAM-1 expression via RAGE, MAPK and NF-кB pathways in human gingival fibroblasts. Journal of Periodontal Research 53 334-344. (https://doi.org/10.1111/ jre.12518) 
Peng Z, Sun Y, Lv X, Zhang H, Liu C \& Dai S 2016 Interleukin-6 levels in women with polycystic ovary syndrome: a systematic review and meta-analysis. PLoS ONE 11 e0148531. (https://doi.org/10.1371/ journal.pone.0148531)

Pertynska-Marczewska M, Diamanti-Kandarakis E, Zhang J \& Merhi Z 2015 Advanced glycation end products: a link between metabolic and endothelial dysfunction in polycystic ovary syndrome? Metabolism: Clinical and Experimental 64 1564-1573. (https://doi.org/10.1016/j. metabol.2015.08.010)

Repaci A, Gambineri A \& Pasquali R 2011 The role of low-grade inflammation in the polycystic ovary syndrome. Molecular and Cellular Endocrinology 335 30-41. (https://doi.org/10.1016/j. mce.2010.08.002)

Roland AV, Nunemaker CS, Keller SR \& Moenter SM 2010 Prenatal androgen exposure programs metabolic dysfunction in female mice. Journal of Endocrinology 207 213-223. (https://doi.org/10.1677/JOE10-0217)

Sathyapalan T, Al-Qaissi A, Kilpatrick ES, Dargham SR \& Atkin SL 2018 Anti-Müllerian hormone measurement for the diagnosis of polycystic ovary syndrome. Clinical Endocrinology 88 258-262. (https://doi. org/10.1111/cen.13517)

Schmidt J, Weijdegård B, Mikkelsen AL, Lindenberg S, Nilsson L \& Brännström M 2014 Differential expression of inflammation related genes in the ovarian stroma and granulosa cells of PCOS women. Molecular Human Reproduction 20 49-58. (https://doi.org/10.1093/ molehr/gat051)

Seki R, Yamagishi S, Matsui T, Yoshida T, Torimura T, Ueno T, Sata M \& Okamura T 2013 Pigment epithelium-derived factor (PEDF) inhibits survival and proliferation of VEGF-exposed multiple myeloma cells through its anti-oxidative properties. Biochemical and Biophysical Research Communications 431 693-697. (https://doi.org/10.1016/j. bbrc.2013.01.057)

Shao H, Schvartz I \& Shaltiel S 2003 Secretion of pigment epitheliumderived factor mutagenic study. European Journal of Biochemistry 270 822-831. (https://doi.org/10.1046/j.1432-1033.2003.03374.x)

Shorakae S, Teede H, de Courten B, Lambert G, Boyle J \& Moran LJ 2015 The emerging role of chronic low-grade inflammation in the pathophysiology of polycystic ovary syndrome. Seminars in Reproductive Medicine 33 257-269. (https://doi. org/10.1055/s-0035-1556568)

Simonovic M, Gettins PG \& Volz K 2001 Crystal structure of human PEDF, a potent anti-angiogenic and neurite growth-promoting factor. PNAS 98 11131-11135. (https://doi.org/10.1073/pnas.211268598)

Skaznik-Wikiel ME, Swindle DC, Allshouse AA, Polotsky AJ \& McManaman JL 2016 High-fat diet causes subfertility and compromised ovarian function independent of obesity in mice.
Obstetrical and Gynecological Survey 71 532-533. (https://doi. org/10.1097/OGX.0000000000000363)

Teede H, Deeks A \& Moran L 2010 Polycystic ovary syndrome: a complex condition with psychological, reproductive and metabolic manifestations that impacts on health across the lifespan. BMC Medicine 8 41. (https://doi.org/10.1186/1741-7015-8-41)

Tombran-Tink J, Chader GG \& Johnson LV 1991 PEDF: a pigment epithelium-derived factor with potent neuronal differentiative activity. Experimental Eye Research 53 411-414. (https://doi. org/10.1016/0014-4835(91)90248-d)

Tombran-Tink J, Mazuruk K, Rodriguez IR, Chung D, Linker T, Englander E \& Chader GJ 1996 Organization, evolutionary conservation, expression and unusual Alu density of the human gene for pigment epithelium-derived factor, a unique neurotrophic serpin. Molecular Vision 211.

Wang JJ, Zhang SX, Lu K, Chen Y, Mott R, Sato S \& Ma JX 2005 Decreased expression of pigment epithelium-derived factor is involved in the pathogenesis of diabetic nephropathy. Diabetes 54 243-250. (https:// doi.org/10.2337/diabetes.54.1.243)

Wang JJ, Zhang SX, Mott R, Chen Y, Knapp RR, Cao W \& Ma JX 2008 Anti-inflammatory effects of pigment epithelium-derived factor in diabetic nephropathy. American Journal of Physiology: Renal Physiology 294 F1166-F1173. (https://doi.org/10.1152/ajprenal.00375.2007)

Yamagishi S \& Imaizumi T 2005 Diabetic vascular complications: pathophysiology, biochemical basis and potential therapeutic strategy. Current Pharmaceutical Design 11 2279-2299. (https://doi. org/10.2174/1381612054367300)

Yamagishi S, Nakamura K, Ueda S, Kato S \& Imaizumi T 2005 Pigment epithelium-derived factor (PEDF) blocks angiotensin II signaling in endothelial cells via suppression of NADPH oxidase: a novel antioxidative mechanism of PEDF. Cell and Tissue Research 320 437-445. (https://doi.org/10.1007/s00441-005-1094-8)

Yamagishi SI, Nakamura K, Matsui T, Yoshida T, Takeuchi M \& Imaizumi T 2007 Pigment epithelium-derived factor (PEDF) blocks advanced glycation end product (AGE)-induced angiogenesis in vitro. Hormone and Metabolic Research 39 233-235. (https://doi.org/10.1055/s-2007-970425)

Yao D \& Brownlee M 2010 Hyperglycemia-induced reactive oxygen species increase expression of the receptor for advanced glycation end products (RAGE) and RAGE ligands. Diabetes 59 249-255. (https://doi. org/10.2337/db09-0801)

Yoshida T, Yamagishi S, Nakamura K, Matsui T, Imaizumi T, Takeuchi M, Koga H, Ueno T \& Sata M 2008 Pigment epithelium-derived factor (PEDF) ameliorates advanced glycation end product (AGE)-induced hepatic insulin resistance in vitro by suppressing Rac-1 activation. Hormone and Metabolic Research 40 620-625. (https://doi.org/10.105 5/s-0028-1083785)

Received in final form 21 September 2019

Accepted 29 October 2019

Accepted Manuscript published online 29 October 2019 https://joe.bioscientifica.com https://doi.org/10.1530/JOE-19-0297
(C) 2020 Society for Endocrinology Published by Bioscientifica Ltd. Printed in Great Britain 